\title{
Untreated schizophrenia in hostels for the homeless: a cause for concern?
}

\author{
M. Marshall, Wellcome Training Fellow; and M. SharPe, Clinical Tutor, University \\ Department of Psychiatry, Warneford Hospital, Oxford OX3 7JX
}

We report two cases from a study of Oxford hostels for the homeless of patients who were suffering from schizophrenia and are not receiving medication. After compulsory admission to hospital and treatment both showed a dramatic improvement in behaviour which was confirmed by a standardised rating scale. We discuss how hostels for the homeless have become both asylums and traps for those who suffer from schizophrenia and conclude that doctors should not under-estimate the potential benefit of anti-psychotic drug therapy even in homeless people with chronic psychosis.

As psychiatric hospitals have closed, hostels for the homeless have had to cope with more sufferers from chronic schizophrenia (Weller et al, 1989; Priest, 1976). The prevalence of mental illness in such hostels is so high that they have been compared to traditional asylums (Baker \& Hall, 1988). A recent study in Oxford (using a behavioural rating scale, REHAB) (Marshall, 1989) highlighted the problem by showing that local hostels were caring for enough severely disabled patients to fill two long-stay wards (48 out of 146 residents) (Timms \& Fry, 1989).

Although superior in some respects to traditional wards, hostels for the homeless do not offer psychiatric treatment. This may not make much difference to those residents who suffer from treatmentresistant 'intrinsic' disabilities, (Leach, 1979) but, as the following case reports demonstrate there is an important minority who benefit greatly from anti-psychotic drugs.

Case One. A 39-year-old man lived in a hostel for seven years. Throughout this time he had been unable to care for himself and refused to bathe. Staff could not communicate with him.

Between the ages of 18 and 25 he suffered five episodes of acute schizophrenia, but each time made a good recovery with anti-psychotic medication. From 25 to 30, while receiving medication, he lived independently and worked for a local charity. Then, after briefly visiting his home town, he lost his flat and moved into an Oxford hostel for the homeless. Around the same time he stopped his medication and relapsed, having refused contact with psychiatric services.

When admitted seven years later to a local psychiatric hospital under Section 2 of the Mental Health
Act, he was flea-infested and suffering from scabies. His thinking was disorganised and he appeared to be hallucinating.

Despite seven years of florid illness and extreme disability, his response to anti-psychotic drugs was dramatic. Within weeks he could wash, dress and hold a normal conversation. He began to look up old friends and make plans for the future. REHAB scores before and after treatment illustrate this improvement. The pre-treatment score of 122 on General Behaviour placed him (in terms of disability) among the worst $10 \%$ of long-stay psychiatric patients. The post-treatment score of 25 indicates a much lower level of disability. He had been the most disabled person in the study of Oxford hostels.

Case Two. A 43-year-old man lived in a hostel for five years, having simply turned up there without belongings. His speech was incomprehensible and nothing was known about his background. He was always dirty and often adopted bizarre postures.

When taken to hospital under Section 2 of the Mental Health Act he appeared thought-disordered and deluded. A diagnosis of schizophrenia was made. After receiving a depot injection of antipsychotic medication he returned to the hostel without any improvement. Over the next few weeks hostel staff observed a change in him. His speech became comprehensible, he started to wash and also shaved for the first time in years. He asked to make contact with relatives in Scotland whom no one had known existed.

His pre-treatment total of 107 on REHAB General Behaviour had made him the fourth most disabled person in the Oxford hostels. Unfortunately he left the hostel before a second rating could be made, but a retrospective rating (based on an interview with his key worker) put his post-treatment score at 20 , a low level of disability.

\section{Comments}

For many patients with "treatment-resistant" schizophrenia, hostels for the homeless are a last refuge in the community. Over the years, through necessity and philanthropy, these units have developed a remarkable capability to tolerate the deviant and support the disabled. But the same qualities 
which make them an asylum for one mentally ill person, can make them a trap for others.

Our case histories illustrate two groups of patients who sometimes become trapped. Members of the first group (exemplified by case one) are fairly socially disabled even at their best, but have a much higher level of functioning when taking medication. Since these patients have poor coping skills, they are always at risk of becoming homeless. If, on entering a hostel, they stop taking medication, the result is a slow deterioration in their condition, so that over time they may become very disabled. Unfortunately their need for treatment can pass unnoticed in an environment where bizarre behaviour is commonplace and accepted.

Members of the second group (of which case two is an example) have tenuous social supports which collapse when they develop psychoses. Instead of being brought to psychiatric attention and receiving appropriate treatment at these times of crisis, they may drift into hostels for the homeless. Here they remain without attracting psychiatric attention, because hostel staff, as a result of their experience with treatment-resistant patients, have learnt to tolerate a level of disturbance which relatives, neighbours or landlords would not. But however good these hostels may be as alternatives to in-patient care, they are not in a position to administer compulsory drug treatment.

To a visiting doctor, chronic patients in hostels for the homeless may all seem much alike. Most residents are socially disabled and "treatment resistant". Attempts to remove them to hospital could be pointless and thankless. However, when the person concerned has not had a trial of medication (and cannot be treated in the community) inactivity is not in the patient's best interest.
We therefore recommend that psychiatrists not only maintain contact with local hostels for the homeless, but also carefully review the treatment history of each severely ill individual. Where a deteriorated person with schizophrenia is not receiving medication, and does not have well documented non-response to such treatment, a trial of an antipsychotic drug should be considered, if necessary admitting the patient to hospital under the Mental Health Act. Subsequent efforts to maintain followup may prevent a repeat of the same pattern of failure to take medication, relapse, social deterioration and failure to receive treatment. These case examples suggest that some of the most "chronic" residents will benefit most from such an active approach to management.

\section{References}

BAKER, R. \& HALl, J. N. (1988) REHAB: A new assessment instrument for chronic psychiatric patients. Schizophrenia Bulletin, 14, 97-111.

LEACH, J. (1979) Providing for the destitute. In Community Care for the Mentally Disabled (eds. WING, J. K. and OLSEN, R.) Oxford: Oxford University Press.

Marshall, M. (1989) Collected and neglected: Are Oxford hostels for the homeless filling up with disabled psychiatric patients? British Medical Journal, 299, 706-709.

Priest, R. G. (1976) The homeless person and the psychiatric services: an Edinburgh study. British Journal of Psychiatry, 10, 233-235.

Timms, P. W. \& FrY, A. H. (1989) Homelessness and mental illness. Health Trends, 21, 70-71.

Weller, M. Tobiansky, R. I., Hollander, D. \& Ibranhimi, S. (1989) Psychosis and destitution at Christmas 1985-1988. Lancet, ii, 1509-1511. 